\title{
GEODESICS ON SURFACES OF GENUS ZERO WITH KNOBS*
}

\author{
BY \\ DONALD EVERETT RICHMOND
}

\section{INTRODUCTION}

Poincaré has studied the geodesics upon closed surfaces of genus zero which are everywhere convex $\dagger$. General surfaces of genus greater than one have been studied by H.M.Morse. $\ddagger$ The surfaces considered in this paper are of genus zero and closed, but have upon them regions of negative curvature as well as regions of positive curvature. They are of such a nature that the removal of certain portions which we call knobs leaves a region with extremalconvex boundaries. A remarkable subset of the geodesics issuing from any point not on a knob is considered, and results are obtained resembling those of Hadamard for geodesics on surfaces of negative curvature. $\$$

\section{Part I. The surface}

1. The surface defined. Let us consider a closed surface homeomorphic with a sphere. We assume that the surface can be divided into a finite number of overlapping regions, such that the cartesian coördinates $x, y, z$ of the points of any of these regions can be expressed in terms of parameters $u$ and $v$ by means of functions with continuous derivatives of at least the fourth order and such that

$$
\left[\frac{D(x, y)}{D(u, v)}\right]^{2}+\left[\frac{D(x, z)}{D(u, v)}\right]^{2}+\left[\frac{D(y, z)}{D(u, v)}\right]^{2} \neq 0 .
$$

It is assumed, moreover, that the surface possesses $n$ knobs $(n>1)$, where a $k n o b$ will be defined as a finite portion $K$ of the surface which is: (a) bounded by a closed curve $C$ with continuously turning tangent; (b) homeomorphic with the interior and boundary points of a circle; (c) such that the geodesics

\footnotetext{
- Presented to the Society, January 2, 1926; received by the editors in August, 1926. p. 237.

† Poincare, Sur les lignes grodesiques des surfaces convexes, these Transactions, vol. 6 (1905),

$\mp$ Morse, $A$ fundamental class of geodesics on any closed surface of genus greater than one, these Transactions, vol. 26 (1924), pp. 49-60.

8 Hadamard, Les surfaces d courbures opposees et leurs lignes geodesiques, Journal de Mathematiques pures et appliquées, (5), vol. 4 (1896), p. 27.
} 
tangent to the boundary $C$ lie interior to $K$ in the immediate neighborhood of the point of contact. Finally, the curves $C$ bounding different knobs on the surface are assumed to have no points in common with each other.

Let now the kncbs be cut from the body of the surface along the curves $C$. There will remain a surface $S$ with $n$ closed bounding curves. $S$ will be extremal-convex in the sense* that, on the original uncut surface, geodesics tangent to the boundary of $S$ lie outside of $S$ in the immediate neighborhood of the point of contact. In this paper we consider geodesics in so far as they lie on $S$.

2. Sufficient conditions for the existence of knobs. If any point $P$ on a regular surface is taken as origin of a system of geodesic polar coördinates, the element of arc takes the form

$$
d s^{2}=d r^{2}+C^{2}(r, \phi) d \phi^{2},
$$

in which $r$ is the distance measured from $P$ along any geodesic through $P$, and $\phi$ is the angle between the tangent at $P$ to this geodesic and that to an arbitrary geodesic through $P$. Also $C(0, \phi)=0$ and $C_{r}(0, \phi)=1$. $\dagger$

Within the region where the geodesics $\phi=$ constant form a field, all the geodesics not $\phi=$ constant are solutions of the Euler equation for

$$
s=\int\left[\dot{r}^{2}+C^{2}(r, \phi)\right]^{1 / 2} d \phi,
$$

namely

$$
\ddot{r}-\left(\frac{2 C_{r}}{C}\right) \dot{r}^{2}-\left(\frac{C_{\phi}}{C}\right) \dot{r}-C C_{r}=0 .
$$

For a geodesic which is tangent to the geodesic circle $r=r_{0}, r=0$ at the point of contact. At this point, therefore,

$$
r=C C_{r} .
$$

In order that the simply connected region bounded by $r=r_{0}$ be a knob it is necessary that for each geodesic tangent to $r=r_{0}, r \leqq 0$ at the point of tangency. A sufficient condition is that $\vec{r}<0$ and hence from (1), it is also sufficient that $C\left(r_{0}, \phi\right)>0$ and $C_{r}\left(r_{0}, \phi\right)<0$ for all values of $\phi$.

We shall deduce a sufficient condition for the existence of $a$ knob in terms of the total curvature.

- Cf. G. D. Birkhoff, Dymamical systems with two degrees of freedom, these Transactions, vol. 18 (1917), p. 216.

† Cf. Darboux, Surfaces, III, p. 157. 
Let any point $P$ on a regular surface be taken as the origin of a system of geodesic polar coördinates $(r, \phi)$ and let $K(r, \phi)$ be the total curvature of the surface at the point $(r, \phi)$.

TheOREM 1. If there exists a constant $r_{0}>0$ such that

$$
\left(\frac{\pi}{2 r_{0}}\right)^{2}<K(r, \phi)<\left(\frac{\pi}{r_{0}}\right)^{2}
$$

for any $\phi$ and for $r \leqq r_{0}$, then the set of points for which $r \leqq r_{0}$ form a knob.

By Gauss's theorem*

$$
C_{r r}(r, \phi)+K(r, \phi) C(r, \phi)=0 .
$$

Consider any value $\phi_{0}$ of $\phi$. We assume that (2) holds for $r \leqq r_{0}$. We shall compare the solutions of the differential equations

$$
\begin{aligned}
& \frac{d^{2} x}{d r^{2}}+\left(\frac{\pi}{2 r_{0}}\right)^{2} x=0, \\
& \frac{\partial^{2} C}{\partial r^{2}}+K(r, \phi) C=0, \\
& \frac{d^{2} z}{d r^{2}}+\left(\frac{\pi}{r_{0}}\right)^{2} z=0,
\end{aligned}
$$

which have the initial conditions

$$
\begin{gathered}
x(0)=0, \frac{d x(0)}{d r}=1 ; \\
C\left(0, \phi_{0}\right)=0, \frac{\partial C\left(0, \phi_{0}\right)}{\partial r}=1 ; \\
z(0)=0, \frac{d z(0)}{d r}=1 .
\end{gathered}
$$

By the use of Sturm's comparison theorem, $\dagger$ it follows from (b) and (c) and the inequality $K<\left(\pi / r_{0}\right)^{2}$, that $C\left(r, \phi_{0}\right)>z(r)>0$ within the interval $0<r \leqq r_{0}$. Similarly, from (a) and (b) and the inequality $\left(\pi / 2 r_{0}\right)^{2}<K$, we have $C\left(r, \phi_{0}\right)<x(r)$ for the same interval. Since $C\left(r, \phi_{0}\right)$ is bounded in the interval, it takes on a maximum for a value $r_{m}$ of $r$ where

$$
0<r_{m} \leqq r_{0} \text {. }
$$

* Cf. W. Blaschke, Vorlesungen über Differential Geometrie, I, p. 61.

† Bieberbach, Theorie der Differentialgleichungen, 1923, pp. 144-5. 
We shall prove that

$$
\frac{r_{0}}{2}<r_{m}<r_{0}
$$

Suppose first that $0<r_{m} \leqq r_{0} / 2$. From (b) and (c), omitting the arguments in the functions concerned, we find readily

$$
C \ddot{z}-z C_{\pi r}=\left[K-\left(\frac{\pi}{r_{0}}\right)^{2}\right] C z .
$$

Integrating with respect to $r$ from $r=0$ to $r=r_{m}$, we have

$$
\left[C \dot{z}-{ }_{z} C_{r}\right]_{0}^{r_{m}}=\int_{0}^{r_{m}}\left[K-\left(\frac{\pi}{r_{0}}\right)^{2}\right] C z d r .
$$

Since $C\left(0, \phi_{0}\right)=z(0)=C_{r}\left(r_{m}\right)=0$,

$$
C\left(r_{m}\right) \dot{z}\left(r_{m}\right)=\int_{0}^{r_{m}}\left[K-\left(\frac{\pi}{r_{0}}\right)^{2}\right] C z d r<0 .
$$

But $C\left(r_{m}\right)>0$ and $\dot{z}\left(r_{m}\right) \geqq 0$ for $r_{m} \leqq r_{0} / 2$. Hence, a contradiction is obtained. Similarly, the assumption $r_{m}=r_{0}$ leads to a contradiction.

Therefore

$$
\frac{r_{0}}{2}<r_{m}<r_{0}
$$

as stated. Hence $C\left(r_{0}, \phi_{0}\right)>0$ and $C_{r}\left(r_{0}, \phi_{0}\right)<0$ and by (1), $r<0$. Since (2) holds for any value $\phi_{0}$ of $\phi$, the theorem follows.

3. Class $A$ geodesic segments. Consider now the surface $S$ and denote the $n$ extremal-convex boundaries by $c_{1}, c_{2}, \cdots, c_{n}$. If $P$ and $Q$ are any two points within or on the boundary of $S$, there exists on $S$ joining $P$ to $Q$ at least one rectifiable curve whose length furnishes a minimum with respect to the lengths of all rectifiable curves on $S$ connecting $P$ and $Q . *$ Such a minimizing arc is a geodesic segment with continuously turning tangent and has no points in common with any boundary $c_{k}$, with the possible exception of $P$ and $Q$ themselves. Such geodesic segments will be called Class $A$ geodesic segments on $S$.

No two class $A$ segments on $S$ not joining the same points can intersect more than once. For suppose two such segments $P Q$ and $R S$ intersect in $D$ and $E$. Then it follows from the definition of geodesics of class $A$ that the segments $D E$ of the two geodesics have the same length. In a portion of $P Q$

- Bolza, Vorlesungen viber Variationsrechnung, 1909, pp. 422, 436. 
including $D E$ as an interior segment, the arc $D E$ can be replaced by its equal segment on $R S$. The resulting curve, however, has corners and hence can be shortened," contrary to the assumption that $P Q$ was a class $A$ segment.

4. The covering surface and linear sets. The surface $S$ can now be rendered simply connected as follows: From any arbitrarily chosen point $P$ on $c_{n}$, we can and will cut the surface along a system of class $A$ geodesics $h_{1}, h_{2}, \cdots, h_{n-1}$, leading to points on $c_{1}, c_{2}, \cdots, c_{n-1}$, respectively. According to the result of the preceding paragraph, the geodesics $h_{k}$ have no other points than $P$ in common. We denote by $T$ the simply-connected piece of surface obtained by cutting $S$ along the $h$ 's.

We now consider $M$, the universal covering surface $\dagger$ of $S$, made up of an infinite number of copies of $T$. On $M$ any two points or curves which overlie the same point or curve on $S$ are said to be congruent. The boundaries of $M$ are congruent to the boundaries $c_{1}, c_{2}, \cdots, c_{n}$ of $S$.

Let $r$ be an integer, positive, negative or zero. Let $T_{r}$ denote a particular copy of $T$ on $M$. A linear set $\ddagger$ of copies of $T$ on $M$ will be defined to be a region of $M$ consisting of a set of the copies of $T$ on $M$ of the form

$$
\cdots, T_{-2}, T_{-1}, T_{0}, T_{1}, T_{2}, \cdots \text {, }
$$

or of the form of any subset of consecutive symbols of (1), in which each copy $T_{k}$ of $T$ is joined to the succeeding one along a common boundary and all copies are distinct. A linear set which has no first or last copy of $T$ will be termed an unending linear set.

\section{Part II. The class $A$ geodesic rays through a poINT}

5. Unending geodesics of class $A$. We have defined (\$3) a geodesic segment joining a pair of points $P$ and $Q$ on $S$ to be of class $A$ on $S$ if its length furnishes a minimum with respect to the lengths of all rectifiable curves on $S$ joining $P$ and $Q$. We shall now define similarly a geodesic segment joining a pair of points $P$ and $Q$ on $M$ to be of class $A$ on $M$ provided its length furnishes a minimum with respect to the lengths of all rectifiable

- In the regular problem of the calculus of variations, the Erdmann corner point condition is never satisfied.

$\dagger$ Cf. H. M. Morse, A one-to-one representation of geodesics on a surface of negative curvature, American Journal of Mathematics, vol. 43 (1921), pp. 35-40; H. Weyl, Die Idee der Riemannschen Fläche, pp. 47-53; Kerékjärt6, Vorlesungen über Topologie, I, pp. 158, 173-184.

‡ For another point of view, cf. Oswald Veblen, Analysis Situs, The Cambridge Colloquium, Part 2, Chapter V. The linear set used in our paper has, however, the important metrical property of being bounded by geodesic segments congruent to the boundaries of $T$, in addition to the abstract properties of the members of the Poincare group. 
curves on $M$ joining $P$ and $Q$. Every geodesic segment of class $A$ on $S$ is also of class $A$ on $M$ but the converse is not true.

Now any two points $P$ and $Q$ on $M$ can be included in a region $R$ consisting of a finite number of copies of $T$. The boundaries of $R$ will be found in part among the boundaries of $M$, composed of segments congruent to the $c_{k}$ 's, and in part among the geodesic segments of class $A$ on $M$ congruent to the $h_{k}$ 's. The segments of the boundary of $R$ congruent to the $c_{k}$ 's intersect the segments congruent to the $h_{k}$ 's so that the angles interior to $R$ do not exceed $\pi$. The region $R$ will still be extremal-convex in the sense of Birkhoff, ${ }^{*}$ and it follows as in $\S 3$ that the points $P$ and $Q$ on $R$ can be joined by a geodesic segment of class $A$ on $M$ which lies in $R$ and has no points in common with the boundary of $R$ with possible exception of $P$ and $Q$ themselves.

We shall now define an unending geodesic of class $A$ on $M$ as an unending geodesic lying entirely on $M$, every finite segment of which is a geodesic segment of class $A$ on $M$.

It follows from a discussion similar to that of $\$ 3$ that no two unending geodesics of class $A$ on $M$ can intersect more than once.

Now let $g$ be an unending geodesic of class $A$ on $M$. Clearly $g$ cannot become infinite in length in any single copy of $T$. In leaving a copy of $T, g$ cannot be tangent to any of the geodesic segments separating that copy of $T$ from the remainder of $M$. Further, $g$ can have only one point of intersection with any such geodesic segment. It follows that an unending class $A$ geodesic on $M$ is contained in one and only one unending linear set.

Henceforth, geodesics and geodesic segments will be assumed to be on $M$ unless the contrary is stated.

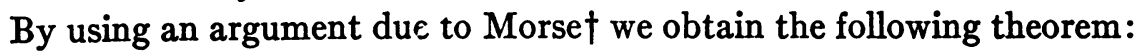

THEOREM 2. Given any unending linear set, there exists at least one unending geodesic of class $A$ contained wholly in the given linear set.

6. Semi-infinite sets and geodesic rays. Let $T_{0}$ be any copy of $T$ on $M$. A semi-infinite linear set starting from $T_{0}$ will be defined by a sequence of copies of $T$ that go to make up $M$, namely: $T_{0}, T_{1}, T_{2}, \cdots$, in which each copy $T_{k}$ of $T$ is joined to the succeeding one along a common boundary and all copies are distinct.

$A$ geodesic ray of class $A$ issuing from a point $P$ will be defined to be a portion of a geodesic, in one sense unending and in the other stopping at $P$, and such that every finite segment is of class $A$. Corresponding to Theorem 2 we have the following theorem for a semi-infinite linear set.

- Loc. cit., p. 216.

$\dagger$ American Journal of Mathematics, loc. cit., pp. 47-48. 
Theorem 3. Given a semi-infinite linear set starting from a copy $T_{0}$, there exists issuing from any point $P$ of $T_{0}$ at least one geodesic ray of class $A$ contained wholly in the given linear set.

If the given surface has two knobs, there are only two semi-infinite linear sets beginning with any copy $T_{0}$ of $T$. If, however, the number of knobs exceeds two, it is readily shown that the number of such sets starting from a given $T_{0}$ has the power of the continuum.

By Theorem 3 there exists issuing from a point $P$ on a given copy $T_{0}$ of $T$ at least one geodesic ray of class $A$ belonging to each semi-infinite linear set beginning with that copy. For $n>2$, therefore, there are through $P$ a set of such geodesic rays in power equal to the power of the continuum.

Let the positive sense of any geodesic ray of class $A$ through $P$ be the sense that leads from $P$. The direction of any such geodesic ray will now be specified by the angle $\theta(-\pi<\theta \leqq \pi)$ measured in an arbitrary sense about $P$, between the positive tangent to it at $P$ and the tangent at $P$ to an arbitrary geodesic through $P$. We shall show that the infinite set of directions $\theta$ has a very remarkable subset which is perfect and nowhere dense.

7. Special and general linear sets. Any semi-infinite linear set $L$ is topographically equivalent to one of the two regions of the plane bounded by two parallel straight lines and a transversal to them. Consider those boundaries $B$ and $B^{\prime}$ of $L$ which in this correspondence are topographically equivalent to the semi-infinite segments of the parallel lines.

Now all the semi-infinite linear sets whose first copy of $T$ contains $P$ will be divided into two classes:

(A) General linear sets;

(B) Special linear sets.

A semi-infinite linear set will be called special if either $B$ or $B^{\prime}$, after at most a finite segment, consists entirely of a boundary of $M$ made up of a succession of segments which are congruent to a single one of the boundaries $c_{k}$ on $S$. Any set not special will be called general.

Consider any two semi-infinite linear sets $L$ and $L^{\prime}$, beginning with $T_{0}$, and let them be represented by the sequences of copies of $T: T_{0}, T_{1}, T_{2}, \cdots$, and $T_{0}, T_{1}^{\prime}, T_{2}^{\prime}, \cdots$, respectively. If the successive copies $T_{1}^{\prime}, T_{2}^{\prime}$, $T_{3}^{\prime}, \cdots, T_{n}^{\prime}$ are respectively the same as $T_{1}, T_{2}, \cdots, T_{n}$, but if $T_{n+1}^{\prime}$ is different from $T_{n+1}$, the two linear sets will be said to diverge after sharing $n$ copies of $T$. Now if $B$ and $B^{\prime}$ are the semi-infinite boundaries of $L$, it is clear that $T_{n+1}^{\prime}$ is joined to $T_{n}^{\prime}=T_{n}$ along a segment $q$ of either $B$ or $B^{\prime}$ which arose from one of the pieces $h_{k}$. The set $L^{\prime}$ will be said to diverge from $L$ along $B$ or $B^{\prime}$ according as $q$ lies on $B$ or $B^{\prime}$. 
The general linear sets (A) and the special linear sets (B) may now be characterized with respect to diverging linear sets as follows: In the case of any general linear set $L$, there exist, corresponding to any positive integer $m$, linear sets which diverge from $L$ along $B$ and along $B^{\prime}$ after sharing with $L$ more than $m$ copies of $T$. In the case of a special linear set $L$, for sufficiently large values of $m$, all linear sets different from $L$ and sharing with $L$ more than $m$ copies of $T$, diverge from $L$ along either $B$ or $B^{\prime}$, but not both.

8. Boundary geodesic rays. Consider now a general linear set $L$. Let $q_{1}, q_{2}, \cdots$ be successive segments of $B$ which arose from cuts $h_{k}$. Let $L_{1}$ be a linear set which diverges from $L$ along $q_{1}, L_{2}$ a linear set which diverges from $L$ along $q_{2}$ and so on. On each linear set $L_{i}$, there exists at least one geodesic ray of class $A$ issuing from $P$. Let $\theta_{1}, \theta_{2}, \cdots$ be the directions at $P$ of geodesic rays of class $A$ issuing from $P$, chosen on $L_{1}, L_{2}, \cdots$, respectively. The set of $\theta$ 's has at least one limit angle $\Theta$. Let $g$ be the geodesic ray through $P$ with the direction $\Theta$. Then $g$ belongs to a linear set, which must be $L$, and is itself of class $A$.

If $\theta$ is measured in a proper sense about $P$, it will be true that for all integers exceeding a suitably chosen integer $m, \theta_{i}<\theta_{i+1}$. Hence $\Theta$ will be the limit of an increasing sequence of the $\theta_{i}$ 's.

It may well happen that there exist in some or all of the linear sets $L_{i}$ more than one geodesic ray of class $A$ issuing from $P$ and belonging to $L_{i}$. It is conceivable that, if a different set of these geodesic rays were picked out, and correspondingly different angles $\theta_{i}{ }^{*}$, a different angle $\Theta^{*}$ would be approached as a limit. If it is remembered, however, that no two geodesic rays issuing from $P$ can intersect, it will be seen that for a proper choice of $\theta=0, \theta_{i-1}<\theta_{i}{ }^{*}<\theta_{i+1}$. Hence $\Theta=\Theta^{*}$ and the geodesic ray determined by the limiting process is unique.

Similarly, let $q_{1}^{\prime}, q_{2}^{\prime}, q_{3}^{\prime}, \cdots$ be successive segments of $B^{\prime}$ which arose from cuts $h_{k}$. Let $L_{1}^{\prime}$ be a linear set diverging from $L$ along $q_{1}^{\prime}$ and so on. If $\theta_{1}^{\prime}, \theta_{2}^{\prime}, \cdots$ are the directions at $P$ of geodesic rays of class $A$ lying on $L_{1}^{\prime}, L_{2}^{\prime}, \cdots$, the set of directions $\theta_{2}^{\prime}$ has a limit $\Theta^{\prime}$ which defines a geodesic ray $g^{\prime}$ through $P$. The geodesic ray $g^{\prime}$ is a ray of class $A$ on $L$ and does not depend upon the particular selection of geodesic rays, issuing from $P$, made from $L_{1}^{\prime}, L_{2}^{\prime}, \ldots$.

The geodesic rays $g$ and $g^{\prime}$ bound a region $R$ in $L$ between which there can lie no geodesic rays of class $A$ issuing from $P$ except those remaining forever in $L$. Further if $g^{\prime \prime}$ be any geodesic ray of class $A$ issuing from $P$ and lying in $L$, and not $g$ or $g^{\prime}$, it must lie in this region $R$. For otherwise $g^{\prime \prime}$ would cut all of the geodesic rays of $L_{i}$ or else all of the geodesic rays of $L_{i}^{\prime}$ which issue from $P$ with angles nearer $\Theta$ or $\Theta^{\prime}$ respectively than the initial angle of $g^{\prime \prime}$. 
The geodesic rays $g$ and $g^{\prime}$ will be called the boundary geodesic rays of the set $L$. In case there is only one geodesic ray of class $A$ on $L, g=g^{\prime}$.

For special linear sets, we adopt a similar procedure except that only one boundary $B$ or $B^{\prime}$ contains an infinite number of segments which arose from cuts $h_{k}$. Hence only one boundary geodesic ray is defined for a special linear set.

We shall consider in the following only the boundary rays of the semiinfinite linear sets whose first copy of $T$ contains $P$.

9. Generalization of a theorem of Hadamard. We prove the following theorem.

TheOREM 4. The set of the directions at $P$ of all the boundary rays of class $A$ issuing from any given point $P$ of $T_{0}$ is perfect and nowhere dense.

It follows from the process by which boundary rays are defined that the direction of each such ray is the limit of the directions of others of the same kind, and further that any limit direction of an infinite subset of directions of boundary rays is itself the direction of a boundary ray. Hence the given set is perfect.

Between the boundary rays of any given linear set (if there are two), there are, of course, geodesic rays with interrnediate directions. Such rays are clearly not themselves boundary rays, since there are at most two boundary rays in any given linear set.

Consider, on the other hand, two boundary rays $g$ and $g^{\prime}$ which belong to different linear sets $L$ and $L^{\prime}$. There exists a last copy of $T$, say $T_{n}$, which the two sets have in common. On the boundary of $T_{n}$, there exists between the pieces to which $T_{n+1}$ of $L$ and $T_{n+1}^{\prime}$ of $L^{\prime}$ are attached, in either cyclic order, at least one point $Q$ on the boundary of $M$. Therefore a class $A$ geodesic segment may be drawn from $P$ to $Q$ whose direction will be between the directions of the boundary rays $g$ and $g^{\prime}$, and which will pass off $M$ at $Q$. All rays through $P$ with angles sufficiently near that of the geodesic segment $P Q$ will also pass off $M$ and hence cannot be boundary rays through $P$. Hence the set of directions of boundary rays through $P$ is nowhere dense and the theorem is proved.

As a special case, this theorem reduces to a result obtained by Hadamard in his study of surfaces of negative curvature. ${ }^{*}$ Let us suppose that the closed surface of genus zero with which we start is such that the removal of the knobs leaves a region $S$ which is of negative curvature throughout. By virtue of this special condition, all geodesic rays issuing from a given point $P$ on $M$

* Loc. cit., p. 69. 
and remaining on $M$ are of class $A$. It can be shown that there exists issuing from $P$, one and only one geodesic ray belonging to each semi-infinite linear set whose first copy of $T$ contains $P$.

The theorem for the special case under consideration may now be expressed in a form not involving the covering surface, and becomes then the theorem of Hadamard:

If $S$ is of negative curvature throughout and if $P$ is any point on $S$, the set of the directions at $P$ of the geodesic rays which issue from $P$ and remain on $S$ is perfect and nowhere dense.

\section{Part III. Periodic geodesics}

10. Definitions. We shall return to the consideration of unending linear sets. Let such a set be represented by the succession of copies of $T$,

$$
\cdots, T_{-2}, T_{-1}, T_{0}, T_{1}, T_{2}, \cdots \text {. }
$$

The symbols of this sequence may be put into one-to-one correspondence with a succession of symbols

$$
\cdots, p_{-2}, p_{-1}, p_{0}, p_{1}, p_{2}, \cdots,
$$

as follows. Let the $2(n-1)$ boundary pieces of $T$, which arise from the cuts $h_{i}(i=1,2, \cdots,(n-1))$, be numbered in cyclic order, beginning with an arbitrary piece. These numbers will then be associated with the boundary pieces of each of the copies $T_{k}$ occurring in (1). In the linear set (1), let $p_{k}$ be the number of the boundary piece of $T_{k}$ to which $T_{k+1}$ is joined. Then (2) is the sequence obtained by replacing $T_{k}$ by $p_{k}$.

Suppose now that (2) is found to consist, in both senses, of an unending repetition of a finite set $G$ of successive symbols. The linear set will then be called periodic and $G$ a generator of the set. Any generator of (2) which is made up of the smallest possible number of symbols will be called a fundamental generator of (2). Any succession of copies of $T$ in (1) which corresponds to a generator of (2) will be called a cycle of the linear set. A fundamental cycle will then be a cycle which corresponds to a fundamental generator.

Those geodesic segments which separate successive cycles of the periodic linear set from each other will clearly be congruent.

In the representation (1) of a given unending linear set $L$, a copy $T_{k}$ of $T$ will be said to occur $n$ copies later than another copy $T_{k}^{\prime}$ of $T$, if $k-k^{\prime}=n$. We shall now define a transformation $t$ of a periodic set $L$ into itself, as follows: Suppose that each fundamental cycle of $L$ is composed of $n$ copies of $T$. Then under $t$ every point of each copy $T_{k}$ of $T$ is to be replaced by its congruent point in that copy of $T$ which in (1) occurs $n$ copies later. Con- 
gruent points under $t$ will be spoken of as congruent points one fundamental cycle apart and congruent points under $t^{m}$ as congruent points $m$ fundamental cycles apart.

11. Lemma on class $A$ geodesic segments joining congruent points. We prove the following lemma.

LEMMa 1. A class $A$ geodesic, joining congruent points $m$ fundamental cycles apart on a periodic linear set, intersects itself on the original surface in such a manner, that if cut at these points of intersection, the resulting geodesic segments can be regrouped, reordered, and rejoined on $M$ so as to make up $m$ curve segments each joining congruent points one fundamental cycle apart.

Let $R$ be the region formed by $m>1$ successive fundamental cycles of a periodic linear set. Let $C$ be a class $A$ geodesic segment connecting congruent points $A$ and $B$ on the boundaries $b_{1}$ and $b_{2}$ of $R$ which separate it from the remainder of the linear set. We shall first prove that there exist on $C$ at least two congruent points one fundamental cycle apart. Let $C^{\prime}$ be the curve segment determined by all the points congruent to $C$ under $t$, the transformation of the preceding paragraph. If $C$ and $C^{\prime}$ have a point in common on $R$, the point in common on $C$, if considered also as a point on $C^{\prime}$, appears clearly congruent to a point on $C$ one fundamental cycle previous. We must show then that $C$ and $C^{\prime}$ do have a point in common on $R$. Now $C$ divides $R$ into two regions $R_{1}$ and $R_{2}$, having no other points in common than those which belong to $C$. We denote by $P_{0}, P_{1}, \cdots, P_{m}$ the intersections of $C$ with the geodesic segments separating different cycles of $R$ from each other and from the remainder of the linear set, the geodesic segments being taken in the order of progression along the set. $C^{\prime}$ does not intersect the first such boundary and extends beyond $R$ in the positive direction of the set. Its intersections with the geodesic boundaries of successive cycles will be denoted by $P_{1}^{\prime}, P_{2}^{\prime}, \cdots, P_{m+1}^{\prime}$.

If $C$ and $C^{\prime}$ have a point in common, the proof is complete. In the contrary case, we assume that $P_{1}^{\prime}$ lies in $R_{2}$ but not on $C$. Then $C^{\prime}$ crosses the boundary of $R_{2}$ either along $C$ or $b_{2}$. In the former case, the proof is again complete. In the later case, the point $P_{m}^{\prime}$ on $b_{2}$ lies in $R_{2}$. Let $s_{1}, s_{2}, \cdots, s_{m}$ be the distances along the geodesic segments separating cycles of the linear set, measured from the points where these segments enter $R_{2}$ from outside the linear set to $P_{1}, P_{2}, \cdots, P_{m}$, respectively. Let $s_{1}^{\prime}, s_{2}^{\prime}, \cdots, s_{m}^{\prime}$ be similarly defined relative to $P_{1}^{\prime}, P_{2}^{\prime}, \cdots, P_{m}^{\prime}$. We have then $s_{m}^{\prime}<s_{m}$, by hypothesis. Since $P_{m}^{\prime}$ is congruent to $P_{m-1}$, we have $s_{m}^{\prime}=s_{m-1}$ and hence $s_{m-1}<s_{m}$. Similarly, $s_{m-1}^{\prime}<s_{m-1}$, whence $s_{m-2}<s_{m-1}$. Proceeding in this manner, we obtain the conditions 


$$
s_{0}<s_{1}<s_{2} \cdots<s_{m-1}<s_{m} \text {, }
$$

as the only possible case under which the proof might fail. But $\boldsymbol{P}_{\boldsymbol{0}}$ and $\boldsymbol{P}_{\mathrm{m}}$ are congruent and therefore $s_{0}=s_{m}$. Hence this set of conditions cannot be satisfied and $C$ and $C^{\prime}$ have a point in common. There exist therefore on $C$ two congruent points one fundamental cycle apart.

Let now $P$ and $Q$ be the points of $C$ one fundamental cycle apart whose existence has just been proved. Let $A P$ and $Q B$ be the segments of $C$ which respectively precede and follow $P Q$ in the order following the linear set. Now consider on $R$ the curve $C_{1}$ which consists of the segment $Q B$ and a segment congruent to $A P$ under the transformation $t$. Then $C_{1}$ joins points on $R$ which are $(m-1)$ fundamental cycles apart and intersects each geodesic segment separating successive cycles once and only once. The procedure of the preceding paragraphs will now show that, if $m>2, C_{1}$ contains a subsegment joining congruent points one cycle apart. Repetition of the process $m$ times gives the conclusion.

12. Existence of periodic geodesics of class $A$. An unending periodic geodesic $g$ is defined as an unending geodesic composed of successive congruent segments. Such a geodesic necessarily lies on an unending periodic linear set $L$, and overhangs a closed geodesic on the surface $S$. A segment of $g$ whose length is that of this closed geodesic on $S$ will be called a fundamental segment of $g$.

Lemma 2. The length of a class $A$ geodesic segment on $M$ is a continuous function of its end points.

Suppose $P Q$ and $P^{\prime} Q^{\prime}$ are two class $A$ geodesic segments such that the distance $P P^{\prime}=e_{1}$ and the distance $Q Q^{\prime}=e_{2}$, where $e_{1}$ and $e_{2}$ are arbitrarily small positive numbers and the distances $P P^{\prime}$ and $Q Q^{\prime}$ are measured along class $A$ geodesic segments. If $d$ and $d^{\prime}$ are respectively the lengths of $P Q$ and $P^{\prime} Q^{\prime}$, it follows from the class $A$ character of $P Q$ that

i.e.,

$$
d \leqq d^{\prime}+\left(e_{1}+e_{2}\right),
$$

$$
d^{\prime} \geqq d-\left(e_{1}+e_{2}\right) .
$$

Likewise from the class $A$ character of $P^{\prime} Q^{\prime}$ it follows that

Combining,

$$
d^{\prime} \leqq d+\left(e_{1}+e_{2}\right) \text {. }
$$

$$
d-\left(e_{1}+e_{2}\right) \leqq d^{\prime} \leqq d+\left(e_{1}+e_{2}\right) \text {. }
$$

The inequalities express the stated continuity property. 
Theorex 5. Corresponding to any unending periodic linear set there exists on the set at least one unending periodic geodesic of class $A$.

Let $D$ be a fundamental cycle of the given linear set. Each pair of congruent points on the boundaries $b_{1}$ and $b_{2}$ separating $D$ from the remainder of the linear set can be joined by a class $A$ geodesic segment lying entirely in $D$. It follows from Lemma 2 that the length of such class $A$ segments is a single-valued continuous function of an end point on either $b_{1}$ or $b_{2}$. Since the domain of such an end point is closed, there exists a segment $A B$ whose length $l_{m}$ gives a minimum among the lengths of all class $A$ geodesics joining congruent points on $b_{1}$ and $b_{2}$. The segment $A B$ will be proved to be a fundamental segment of a periodic geodesic of class $A$.

Choose arbitrarily a positive direction along $b_{1}$ and a corresponding congruent positive direction along $b_{2}$. Then $A B$ makes the same angle with $b_{1}$ as it does with $b_{2}$. Otherwise, on the surface obtained by "healing" $b_{1}$ and $b_{2}$ together, $A B$ would have a corner and could be shortened on this surface. Recutting the surface to form $D$, the shortened curve would connect congruent points on $b_{1}$ and $b_{2}$. The class $A$ geodesic segment joining these same two congruent points would be shorter than $A B$, contrary to the supposition that $A B$ was the shortest class $A$ segment connecting congruent points on $b_{1}$ and $b_{2}$.

It now follows that in the given unending periodic set of which $D$ is a fundamental cycle, the curves congruent to $A B$ in the successive cycles join on to each other continuously to form an unending periodic geodesic $g$ of which $A B$ is a fundamental segment.

We shall prove by the aid of Lemma 1 that $g$ is of class $A$.

Assume, on the contrary, that there exist on $g$ two points $P$ and $Q$ which can be connected on $M$ by a curve shorter than the segment $P Q$ of $g$. Then there can be found on $g$ two points $A$ and $B, m$ fundamental cycles apart, containing $P$ and $Q$ between them and lying on geodesic segments separating cycles of the linear set from each other, where $m$ is a properly chosen positive integer.

On $g$, the segment $A B$ has the length $m l_{m}$. Now join $A$ and $B$ by a geodesic segment $C$ of class $A$ and length $C$. By hypothesis, $C<m l_{m}$. Hence at least one of the $m$ segments of $C$ of Lemma 1 is necessarily less than $l_{m}$ in length. It would follow that a pair of congruent points could be found on the geodesic boundaries of a fundamental cycle, for which the shortest connecting path on $M$ would be in length less than $l_{m}$, contrary to the definition of $l_{m}$. Therefore $g$ is of class $A$ and the theorem is completely proved.

It follows from the theorem just proved that there exist on an extremal- 
convex surface $S$ with at least three boundaries, an enumerably infinite number of closed (or periodic) geodesics. In particular, there exist closed geodesics deformable into each of the boundaries of $S$.

13. Class $A$ geodesics of the same type. In the remainder of the paper we will state without proof the consequences of the supposition that there exist two or more class $A$ periodic geodesics of the same type, that is, belonging to the same linear set.

It is necessary that all such periodic geodesics of the same type have fundamental segments of the same length.

No two class $A$ periodic geodesics, $g_{1}$ and $g_{2}$, of the same type can intersect, for if they intersected once, they would intersect an infinite number of times, contrary to a fundamental property of class $A$ geodesics. Hence $g_{1}$ and $g_{2}$ separate a ribbon-like region $R$ from the remainder of the set. We suppose that on $R$ there exist no other periodic geodesics of class $A$.

TheORem 6. If a class $A$ geodesic g lies completely on a region $R$ bounded by two class $A$ periodic geodesics $g_{1}$ and $g_{2}$ and there exist on $R$ no class $A$ periodic geodesics other than $g_{1}$ and $g_{2}$, then $g$ is either asymptotic to $g_{1}$ in its positive sense and to $g_{2}$ in its negative sense or it is asymptotic to $g_{2}$ in its positive sense and $g_{1}$ in its negative sense, where the positive sense of $g$ is taken to be that sense which follows the linear set to which $g$ belongs.

The results for geodesic rays are similar.

TheORem 7. There exist issuing from any point $P$ between $g_{1}$ and $g_{2}$ at least four geodesic rays of class $A$, respectively

(a) positively asymptotic to $g_{1}$,

(b) negatively asymptotic to $g_{1}$,

(c) positively asymptotic to $g_{2}$,

(d) negatively asymptotic to $g_{2}$.

Moreover, any geodesic ray of class $A$ issuing from $P$ belongs to one of these four classes.

Cornell Universtry, ITHACA, N. Y. 